\title{
ON THE NUMBER OF LOCALLY BOUNDED FIELD TOPOLOGIES
}

\author{
JO-ANN D. COHEN
}

\begin{abstract}
Kiltinen has proven that there exist $2^{|F|}$ first countable, locally bounded field topologies (the maximum number possible) on a field $F$ of infinite transcendence degree over its prime subfield. We consider those fields $F$ of countable transcendence degree over its prime subfield $E$. In particular it is shown that if the characteristic of $F$ is zero and the transcendence degree of $F$ over $E$ is nonzero or if $F$ is a field of prime characteristic and the transcendence degree of $F$ over $E$ is greater than one, then there exist $2^{|F|}$ normable, locally bounded field topologies on $F$.
\end{abstract}

1. Introduction and basic definitions. Let $R$ be a ring and let $\sigma$ be a ring topology on $R$, that is, $\mathcal{T}$ is a topology on $R$ making $(x, y) \rightarrow x-y$ and $(x, y) \rightarrow x y$ continuous from $R \times R$ to $R$. A subset $A$ of $R$ is bounded for $\sigma$ if given any neighborhood $U$ of zero, there exists a neighborhood $V$ of zero such that $(V A) \cup$ $(A V) \subseteq U . \mathcal{T}$ is a locally bounded topology on $R$ if there exists a fundamental system of neighborhoods of zero for $\mathcal{T}$ consisting of bounded sets.

We recall that a norm $N$ on a ring $R$ is a function from $R$ into the nonnegative reals satisfying $N(x)=0$ if and only if $x=0, N(x-y) \leqslant N(x)+N(y)$ and $N(x y) \leqslant N(x) N(y)$ for all $x$ and $y$ in $R$. If $N$ is a norm on $R$, then $\left\{B_{\varepsilon}: \varepsilon>0\right\}$ is a fundamental system of neighborhoods of zero for a Hausdorff, locally bounded topology $\widetilde{T}_{N}$ on $R$ where for each $\varepsilon>0, B_{\varepsilon}=\{r \in R: N(r)<\varepsilon\}$. Two norms on $R$ are equivalent if they define the same topology on $R$.

If $N$ is a nontrivial norm on a field $F$, that is, $\sigma_{N}$ is nondiscrete, then a subset $A$ of $F$ is bounded for $\mathcal{T}_{N}$ if and only if $A$ is bounded in norm. Furthermore, if $N$ is a nontrivial norm on $F$, then $\mathcal{T}_{N}$ is a field topology on $F$, that is, $\mathcal{T}_{N}$ is a ring topology on $F$ and the mapping $x \rightarrow x^{-1}$ from $F^{*}$ to $F^{*}$ is continuous as well. (The proof of this assertion is the same as that for normed algebras found on p. 75 of [1].) We shall make use of the following theorem proved by Cohn in [3, Theorem 6.1]: If $\mathcal{T}$ is a Hausdorff locally bounded ring topology on a field $F$ and if there exists a nonzero element $c$ in $F$ such that $\lim _{n \rightarrow \infty} c^{n}=0$, then $\mathcal{T}$ is normáble. Hence by the previous remarks, $\mathcal{T}$ is a Hausdorff, first countable, locally bounded field topology on $F$.

If $F$ is any field, then there exist at most $2^{|F|}$ locally bounded ring topologies on $F$ [6, Theorems 5 and 6]. In [5, proof of Theorem 2.1], Kiltinen proved that if $F$ is a field of infinite transcendence degree over its prime subfield, then there exist $2^{|F|}$ first countable, locally bounded field topologies on $F$, the maximum number possible.

Received by the editors December 14, 1982.

1980 Mathematics Subject Classification. Primary 12J99; Secondary 13J99. 
The problem of determining the number of first countable locally bounded field topologies on a field $F$ of finite transcendence degree over its prime subfield was first raised by Kiltinen in [5, p. 35] and again by Wieslaw in [9, p. 175]. In this paper we consider those fields $F$ of countable transcendence degree over its prime subfield $E$. In particular it is shown that if the characteristic of $F$ is zero and the transcendence degree of $F$ over $E$ is nonzero or if $F$ is a field of prime characteristic and the transcendence degree of $F$ over $E$ is greater than one, then there exist $2^{|F|}$ normable, locally bounded field topologies on $F$.

\section{Locally bounded field topologies.}

Lemma 1. Let $X$ be a set of cardinality $\aleph_{0}$. Then there exists a collection $\mathbb{Q}$ of subsets of $X$ satisfying:

1. $|\mathcal{Q}|=2^{\aleph_{0}}$,

2. if $E \in \mathbb{Q}$, then $|E|=\boldsymbol{\aleph}_{0}$,

3. if $E_{1}$ and $E_{2}$ are distinct elements of $\mathcal{Q}$, then $\left|E_{1} \backslash E_{2}\right|=\aleph_{0}$, and $\left|E_{2} \backslash E_{1}\right|=\aleph_{0}$.

Proof. We may assume that $X$ is the set of rational numbers. For each irrational number $y$, let $\left\langle r_{i}\right\rangle_{i=1}^{\infty}$ be a sequence of rational numbers converging to $y$ in the usual topology on the reals and let $E_{y}=\left\{r_{i}: i=1,2, \ldots\right\}$. If $y \neq z$, then $E_{y} \cap E_{z}$ is finite. Hence the set $\mathcal{Q}$, defined by $\mathcal{Q}=\left\{E_{y}: y\right.$ irrational $\}$, satisfies properties $1-3$.

(The author is grateful to Richard Hodel for simplifying her proof of Lemma 1.)

Let $F$ be an infinite field and let $\left\langle F_{n}\right\rangle_{n=0}^{\infty}$ be a sequence of subrings of $F .\left\langle F_{n}\right\rangle_{n=0}^{\infty}$ is a decomposition of $F$ if $1 \in F_{0}, F_{n}$ is properly contained in $F_{n+1}$ for all $n \geqslant 0$ and $F=\cup_{n=0}^{\infty} F_{n}$. Let $\mathscr{Q}$ be a collection of decompositions of $F$. Define $\sim$ on $\mathscr{Q}$ by $\left\langle F_{n}\right\rangle_{n=0}^{\infty} \sim\left\langle G_{n}\right\rangle_{n=0}^{\infty}$ if for each countable subset $A$ of $F, A \subseteq F_{N}$ for some $N \geqslant 0$ if and only if $A \subseteq G_{M}$ for some $M \geqslant 0$. Clearly, $\sim$ is an equivalence relation on $\mathscr{Q}$.

LEMMA 2. Let $F$ be a field and let $E$ be its prime subfield. If the characteristic of $F$ is zero or if the transcendence degree of $F$ over $E$ is nonzero, then there exists a collection Q of pairwise inequivalent decompositions of $F$ such that $|\mathcal{Q}|=2^{\aleph_{0}}$.

Proof. Suppose the transcendence degree of $F$ over $E$ is nonzero. Then there exists a subfield $E_{0}$ of $F$ and a transcendental element $x$ over $E_{0}$ such that $F$ is an algebraic extension of $E_{0}(x)$. Let $p_{1}, p_{2}, \ldots$ be a sequence of pairwise nonassociate, irreducible elements of $E_{0}[x]$ and for each $i \geqslant 1$, let $\hat{v}_{p_{i}}$ be an extension of the $p_{i}$-adic valuation from $E_{0}(x)$ to $F$. Let $A=\left\{p_{A, 0}, p_{A, 1}, \ldots\right\}$ be any countably infinite subset of $\left\{p_{1}, p_{2}, \ldots\right\}$. For each $n \geqslant 0$, let $F_{A, n}=\left\{a \in F: \hat{v}_{p_{A, i}}(a) \geqslant 0\right.$ for all $\left.i \geqslant n\right\}$. Clearly, $1 \in F_{A, 0}, F_{A, n}$ is a subring of $F$ for all $n \geqslant 0$ and $F_{n}$ is properly contained in $F_{n+1}$ for all $n \geqslant 0$ as $p_{A, n}^{-1} \in F_{n+1} \backslash F_{n}$. Moreover, $F=\cup_{n=0}^{\infty} F_{A, n}$. So $\left\langle F_{A, n}\right\rangle_{n=0}^{\infty}$ is a decomposition of $F$. Let $Q$ be a collection of subsets of $\left\{p_{1}, p_{2}, \ldots\right\}$ satisfying properties 1-3 of Lemma 1. If $A$ and $B$ are distinct element of $\mathcal{Q}$ and $A \backslash B=\left\{q_{i}\right.$ : $i=1,2, \ldots\}$, then $\left\{q_{i}^{-1}: i=1,2, \ldots\right\} \subseteq F_{B, 0}$ but $\left\{q_{i}^{-1}: i=1,2, \ldots\right\}$ is not contained in $F_{A, n}$ for any $n \geqslant 0$. Thus $\left\langle F_{A, n}\right\rangle_{n=0}^{\infty}$ and $\left\langle F_{B, n}\right\rangle_{n=0}^{\infty}$ are inequivalent decompositions of $F$. 
If the transcendence degree of $F$ over $E$ is zero, then $F$ is a field of characteristic zero. Let $p_{1}, p_{2}, \ldots$ be a sequence of distinct, positive primes in $\mathbf{Z}$ and proceed as above.

Let $F$ be an infinite field, let $\left\langle F_{n}\right\rangle_{n=0}^{\infty}$ be a decomposition of $F$ and let $x$ be a transcendental element over $F$. We may identify $F(x)$ with a subfield of the field of formal power series $F((x))$ over $F$. Define $\phi: F \rightarrow \mathbf{N} \cup\{0\}$ by $\phi(a)$ is the smallest nonnegative integer $n$ such that $a \in F_{n}$. Define $|\cdot|: F \rightarrow \mathbf{N} \cup\{0\}$ by

$$
|a|= \begin{cases}2^{\phi(a)} & \text { if } a \neq 0 \\ 0 & \text { if } a=0 .\end{cases}
$$

Let $D=\left\{\sum a_{i} x^{i} \in F((x)): \lim _{i \rightarrow \infty}\left|a_{i}\right| 2^{-i}=0\right\}$ and for each $\sum a_{i} x^{i}$ in $D$, let $N\left(\sum a_{i} x^{i}\right)=\sup _{i}\left|a_{i}\right| 2^{-i}$. Then $D$ is a subfield of $F((x)), N$ is a norm on $D$ and $D$ is the completion of $F(x)$ for the $N$-topology [2, Lemmas 2 and 3].

LEMma 3. Let $x$ be a transcendental element over an infinite field $F$. If $\left\langle F_{1, n}\right\rangle_{n=0}^{\infty}$ and $\left\langle F_{2, n}\right\rangle_{n=0}^{\infty}$ are inequivalent decompositions of $F$, then there exist distinct, nondiscrete, normable locally bounded field topologies $T_{1}$ and $T_{2}$ on $F(x)$ corresponding to $\left\langle F_{1, n}\right\rangle_{n=0}^{\infty}$ and $\left\langle F_{2, n}\right\rangle_{n=0}^{\infty}$ respectively.

Proof. By the above remarks, there exist norms $N_{1}$ and $N_{2}$ on $F(x)$ corresponding to the decompositions $\left\langle F_{1, n}\right\rangle_{n=0}^{\infty}$ and $\left\langle F_{2, n}\right\rangle_{n=0}^{\infty}$, respectively. Let $A$ be a subset of $F$ such that $A \subseteq F_{1, n}$ for some $n \geqslant 0$ but $A \nsubseteq F_{2, m}$ for any $m \geqslant 0$. Then $A$ is bounded in norm for $N_{1}$ but not for $N_{2}$. Consequently, the topologies defined on $F(x)$ by $N_{1}$ and $N_{2}$ are distinct.

THEOREM 1. Let $F$ be a field of characteristic zero, let $E$ be its prime subfield and let $\Im$ be a transcendence base for $F$ over $E$.

1.If $\beta=\phi$ and $[F: E]<\infty$, then there exist $\boldsymbol{\kappa}_{0}$ first countable, locally bounded field topologies on F. Moreover each nondiscrete, Hausdorff, locally bounded field topology on $F$ is normable and hence is first countable.

2. If $|\Re|$ is countable and nonzero, then there exist $2^{N_{0}}$ normable, locally bounded field topologies on $F$.

Proof. 1 follows from Theorems 1.8 and 3.3 of [7]. We may therefore assume that $|\Re|$ is nonzero and countable. Thus $|F|=\boldsymbol{\aleph}_{0}$ and so there exist at most $2^{\boldsymbol{\kappa}_{0}}$ locally bounded ring topologies on $F$. Moreover, there exists a subfield $E_{0}$ of $F$ and a transcendental element $x$ over $E_{0}$ such that $F$ is an algebraic extension of $E_{0}(x)$. By Lemmas 2 and 3 , there exist $2^{\aleph_{0}}$ distinct, normable topologies on $E_{0}(x)$. By [8, Theorem 1.6], each locally bounded ring topology on $E_{0}(x)$ extends to a locally bounded ring topology on $F$. But if $\mathcal{T}$ is a locally bounded ring topology on $F$ whose restriction to $E_{0}(x)$ is defined by a nontrivial norm, then there exists a nonzero element $c$ in $E_{0}(x)$ such that $c^{n} \rightarrow 0$ for $\mathcal{T}$. Thus by Cohn's Theorem [3, Theorem 6.1], $\mathcal{T}$ is normable and hence $\mathcal{T}$ is a locally bounded field topology on $F$. 
THEOREM 2. Let $F$ be a field of prime characteristic, let $E$ be its prime subfield and let $\mathscr{G}$ be a transcendence base for $F$ over $E$.

1. If $\mathscr{B}=\phi$, then there exist two first countable, locally bounded field topologies on $F$.

2. If $|\Re|=1$ and $[F: E(\mathscr{B})]<\infty$, then there exist $\boldsymbol{\aleph}_{0}$ first countable, locally bounded field topologies on $F$. Moreover each nondiscrete, Hausdorff, locally bounded field topology on $F$ is normable and hence first countable.

3. If $|\Re|$ is countable and greater than one, then there exist $2^{\aleph_{0}}$ normable, locally bounded field topologies on $F$.

Proof. By [4, Theorem 6.1], if $F$ is an algebraic extension of $E$, then the only locally bounded ring topologies on $F$ are the discrete and indiscrete topologies. The proof of 2 is the same as the proof of 1 of Theorem 1 . If $|\Re| \geqslant 2$, let $x_{1} \in \mathscr{B}$ and let $E_{0}=E\left(\mathscr{B} \backslash\left\{x_{1}\right\}\right)$. Then the transcendence degree of $E_{0}$ over $E$ is nonzero. The proof that there exist $2^{\aleph_{0}}$ normable, locally bounded field topologies on $F$ is the same as the proof of 2 of Theorem 1.

\section{REFERENCES}

1. N. Bourbaki, Topologie générale, Chapitre 9, Hermann, Paris, 1958.

2. J. Cohen, Norms on $F(X)$, Pacific J. Math. (to appear).

3. P. M. Cohn, An invariant characterization of pseudo-valuations on a field, Math. Proc. Cambridge Philos. Soc. 50 (1954), 159-177.

4. J. Kiltinen, Inductive ring topologies, Trans. Amer. Math. Soc. 134 (1968), 149-169.

5. . On the number of field topologies on an infinite field, Proc. Amer. Math. Soc. 40 (1973), $30-36$.

6. H.-J. Kowalsky and H. Dürbaum, Arithmetische Kennzeichung von Körpertopologien, J. Reine Angew. Math. 191 (1953), 135-152.

7. H. Weber, Charakterisierung der lokalbeschränkten Ringtopologien auf globalen Körpern, Math. Ann. 239 (1979), 193-205.

8. Topologische Charakterisierung globaler Körper und algebraischer Funktionenkörper in einer Variablen, Math. Z. 169 (1979), 167-177.

9. W. Wieslaw, Topological fields, Acta Univ. Wratislav. Mat. Fiz. Astronom., No. 675, Wroclaw, 1982.

Department of Mathematics, North Carolina State University, Raleigh, North Carolina 27650 